\title{
EIGENVALUE DECAY OF INTEGRAL OPERATORS GENERATED BY POWER SERIES-LIKE KERNELS
}

\author{
D. Azevedo And V. A. Menegatto
}

Abstract. We deduce decay rates for eigenvalues of integral operators generated by power serieslike kernels on a subset $X$ of either $\mathbb{R}^{q}$ or $\mathbb{C}^{q}$. A power series-like kernel is a Mercer kernel having a series expansion based on an orthogonal family $\left\{f_{\alpha}\right\}_{\alpha \in \mathbb{Z}_{+}^{q}}$ in $L^{2}(X, \mu)$, in which $\mu$ is a complete measure on $X$. As so, we show that the eigenvalues of the integral operators are given by an explicit formula defined by the coefficients in the series expansion of the kernel and the elements of the orthogonal family. The inequalities and, in particular, the decay rates for the sequence of eigenvalues are obtained from decay assumptions on the sequence of coefficients in the expansion of the kernel and on the sequence $\left\{\left\|f_{\alpha}\right\|\right\}_{\alpha \in \mathbb{Z}_{+}^{q}}$.

Mathematics subject classification (2010): 45P05, 32A05, 47B65, 42C05.

Keywords and phrases: Eigenvalue inequalities, eigenvalue decay, power series expansions, positive definite kernels, orthogonal family.

\section{REFERENCES}

[1] M. H. Castro, V. A. Menegatto, Eigenvalue decay of positive integral operators on the sphere, Math. Comput., 81 280, p. 2303-2317, (2012).

[2] E. W. Cheney, Analysis for applied mathematics, Graduate Texts in Mathematics, 208, SpringerVerlag, New York, (2001).

[3] C. M. Dikmen And J. B. Reade, Factorisation of positive definite operators, Arch. Math., 91 4, p. 339-343, (2008).

[4] M. Dostanic, Asymptotic behavior of eigenvalues of certain integral operators Publ. Inst. Math. (Beograd) (N.S.), 59 73, p. 95-113, (1996).

[5] J. C. Ferreira, V. A. Menegatto, Eigenvalues of integral operators defined by smooth positive definite kernels, Integral Equations Operator Theory, 64, 1, p. 61-81, (2009).

[6] J. C. Ferreira, V. A. Menegatto, A. P. Peron, Integral operators on the sphere generated by positive definite smooth kernels, J. Complexity, 24, 5-6, p. 632-647, (2008).

[7] G. B. Folland, Real analysis. Modern techniques and their applications, second edition, Pure and Applied Mathematics (New York), A Wiley-Interscience Publication, John Wiley \& Sons, Inc., New York, (1999).

[8] I. C. Gohberg, M. G. Krein, Introduction to the theory of linear nonselfadjoint operators, translated from the Russian by A. Feinstein, translations of Mathematical Monographs, Vol. 18, American Mathematical Society, (1969).

[9] C.-W. HA, Eigenvalues of differentiable positive definite kernels, SIAM J. Math. Anal., 17 2, p. 415 419, (1986).

[10] H. KÖNIG, Eigenvalue distribution of compact operators, Operator Theory: Advances and Applications, 16, Birkhäuser Verlag, (1986).

[11] B. D. KotluaR, Singular numbers of integral operators, (Russian) Differentsial'nye Uravneniya, 14 8, p. 1473-1477, (1978).

[12] J. B. Reade, Eigenvalues of Lipschitz kernels, Math. Proc. Camb. Phil. Soc., 93 1, p. 135-140, (1983).

[13] W. Rudin, Function theory in the unit ball of $\mathbb{C}^{n}$, Reprint of the 1980 edition, Classics in Mathematics Springer-Verlag, Berlin, (2008). 
[14] Y. SOYKAn, G. LitTLE, Eigenvalues of positive integral operators with Laplace transform-type kernels, Glasg. Math. J., 52 2, p. 333-348, (2010).

[15] KeHE ZHU, Spaces of holomorphic functions in the unit ball, Graduate Texts in Mathematics, 226, Springer-Verlag, New York, (2005). 